
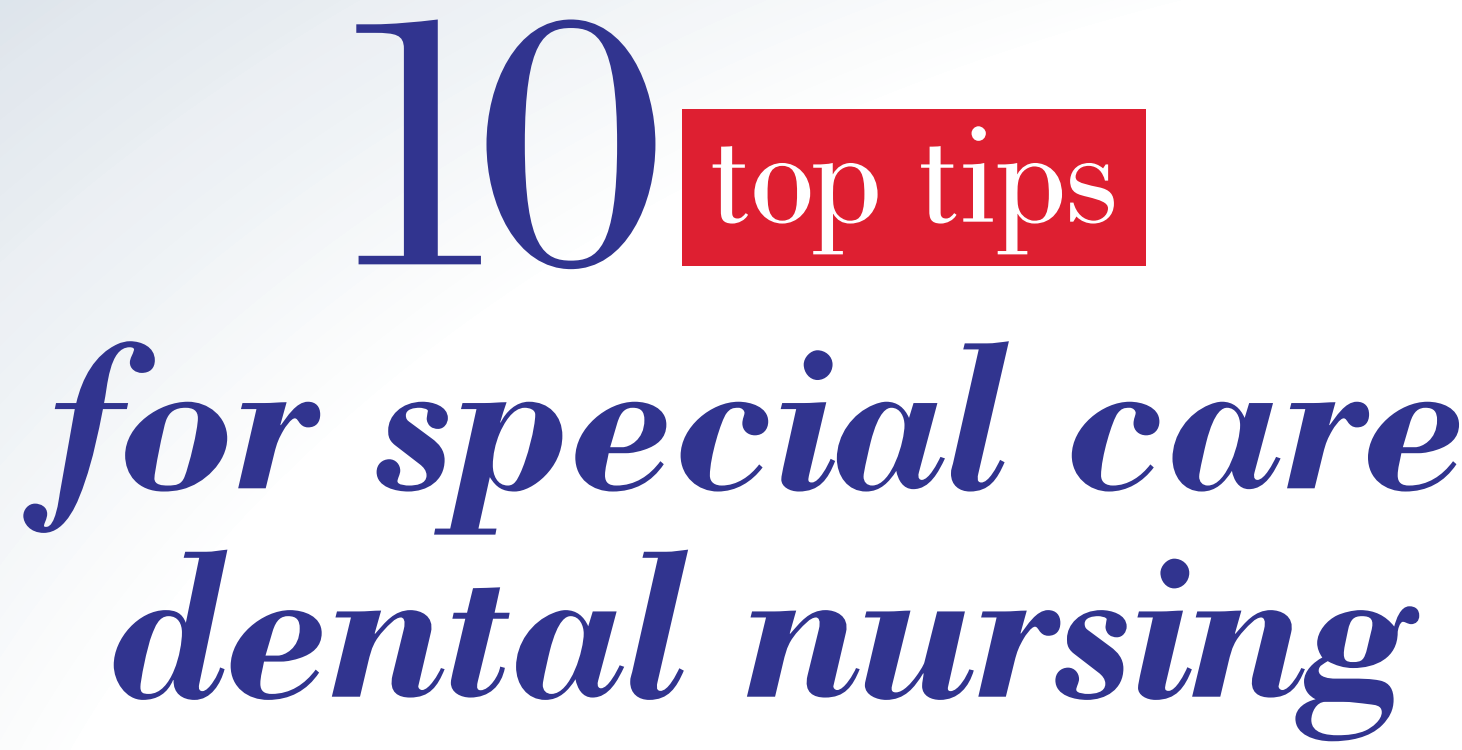

Patients with additional needs may require you to give additional thought into how they access your service. All patients have individual needs specific only to them so care must be tailored to each patient. Dental Nurse Nicola

Sherlock thinks the following ten top tips may help the dental team when treating some of the many patients with more complex needs.

1

\section{Get information}

Garner as much information about the patient and their social/medical history as possible prior to their first appointment Asking some of the following questions will really help you gain a better understanding of the patient:

- Do they have speech, sight or hearing difficulties?

- Do they usually consent for treatment themselves?

- Who will they attend with or will they attend alone?

- Do they use a wheelchair or another mobility aid?

- Does the patient become agitated in unfamiliar surroundings?

- Are they known to lash out or bite when feeling threatened or anxious?

- Do they demonstrate Pica (eating non-food items such as stones, paperclips)? If so, all reception and surgery surface areas must be clear of any small objects prior to the patient's visit.
All information gathered can help to tailor the environment and make their first visit as smooth as possible as this first trip is crucial, particularly for patients with learning disabilities or dental anxieties.

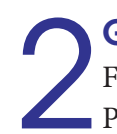

\section{Give Information}

Fore-warned is fore-armed.

Patients worry about coming to

the dentist. Patients with additional needs and their family are no different, throw into the mix a genuine dental phobia and we're guaranteed a failed appointment.

Give directions to the clinic; tell them the best place to park if extra space is required to unpack a wheelchair.

Tell them what to expect on the first visit. Often it is just a chat about medical history and treatment plans but the patient may be envisaging extractions under general anaesthetic (GA).

Inform the patient's parents or care team that they should bring along their medical 
history information, a list of medication, rescue medications if needed, funds for payment or proof of exemptions. If the patient has a personal information booklet or folder (a 'Traffic Light Passport') which details all of the above, ask them to bring it.

It will always help if you tell them a little about your practice. Simply having the name of the dental nurse who will be at their first visit can be a comfort as they have a point of contact.

\section{Identify barriers to care \\ 3 In an ideal world we would all have purpose-built, ground floor facilities} with wheelchair access, dropped kerbs and car parking in close proximity to the building for ease of access but if you don't have that clinic you need to work as a team to answer questions such as:

- How can you facilitate the patient's visit?

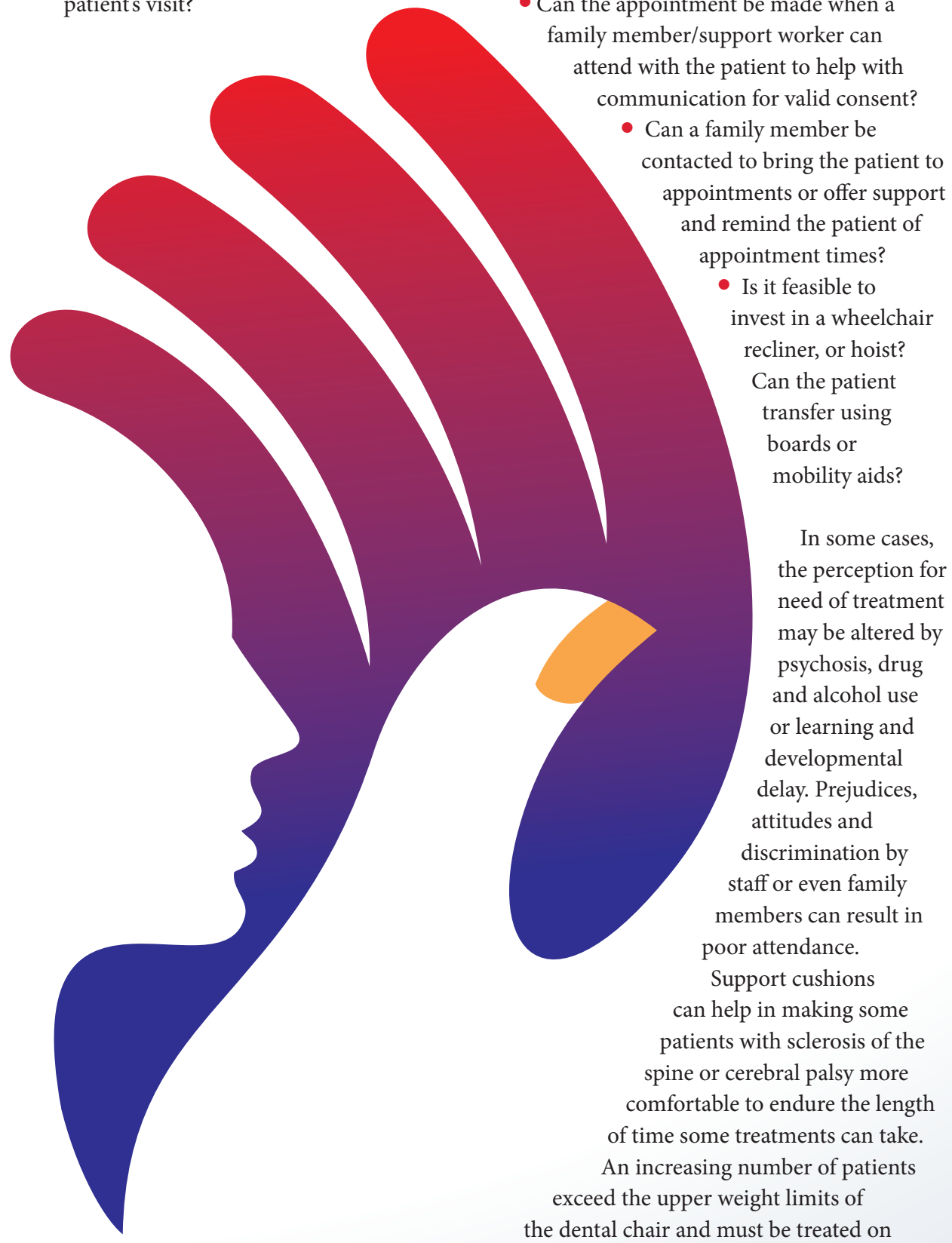

- Could one of the team meet the patient outside and help to guide them into the clinic?

- Can you offer a domiciliary (home) visit or refer them to another service provider who can?

Can the appointment be made when a family member/support worker can cond with the patient to help with

- Can a family member be ntacted to bring the patient to pointments or offer support remind the patient of nvest in a wheelchair recliner, or hoist? Can the patient ransfer using boards or In some cases, the perception for need of treatment be altered by psychosis, drug and alcohol use or learning and developmental delay. Prejudices, staff or even family members can result in oor attendance. spine or cerebral palsy more comfortable to endure the length fime some treatments can take. the dental chair and must be treated on

\section{'SUPPORT CUSHIONS CAN HELP IN MAKING} SOME PATIENTS WITH SCLEROSIS OF THE

SPINE OR CEREBRAL PALSY MORE COMFORTABLE'

a Bariatric chair or plinth. It would be important to obtain details of the patient's weight and when they were last weighed prior to their appointment or when referring said patients to a hospital or community dental service.

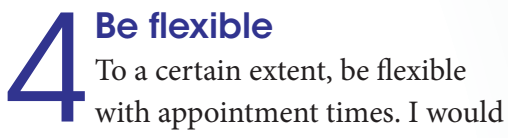
recommend offering an afternoon appointment if the patient has mobility difficulties such as Multiple Sclerosis and requires time in the morning to wait for carers to help them dress, wash, or take their medication. A patient with a neurological disease such as Parkinson's which requires them to wait a certain amount of time after taking their medications in order to function without severe symptoms may also need an afternoon appointment.

Some medications may affect the patient's ability to co-operate with dental treatment. Similarly, if a patient is an alcoholic or drug abuser they may require a morning appointment before they are too selfmedicated to co-operate with care.

Some patients get anxious or agitated if they have to hang around in the waiting room (notably those with autistic spectrum); the first appointment of the session is advisable in these instances as there (in theory) will not be a back-log of patients before them.

Be aware that a patient's health may dictate their ability to attend appointments and may affect their overall attendance; some leniency in rescheduling must be afforded.

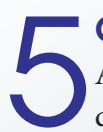

\section{Consent}

A person's ability to make an informed decision resulting in 'valid consent' can be affected for many different reasons such as dementia, learning difficulties, brain injury or mental illness.

As it states in the GDC Standards for the Dental Team: 
You must always consider whether patients are able to make decisions about their care themselves, and avoid making assumptions about a patient's ability to give consent. This is a complex area and you should refer to the appropriate legislation.

If an adult patient is assessed as lacking the capacity to give valid consent a 'Best Interest Decision' may be required at some point whereby the patient, staff, care managers and an Independent Mental Capacity Advocate (IMCA) meet to establish a treatment plan (or not) which is in the patient's best interest. This is a legal right for people over 16 who
7

\section{Anxious patients}

Many patients are not keen on a trip to the dentist but it can often be easy to spot the terror a true dental-phobe experiences. Acknowledge their anxiety. Show empathy in all forms of communication including body language. I would recommend you think SOFTEN: Smile, Open posture, Forward lean, Touch, Eye contact, Nod.

Give the patient control techniques such as phrases or actions. Raising their hand if they want to stop or keep the surgery door ajar have worked in the past. Offer reminders prior to appointments to ensure attendance.

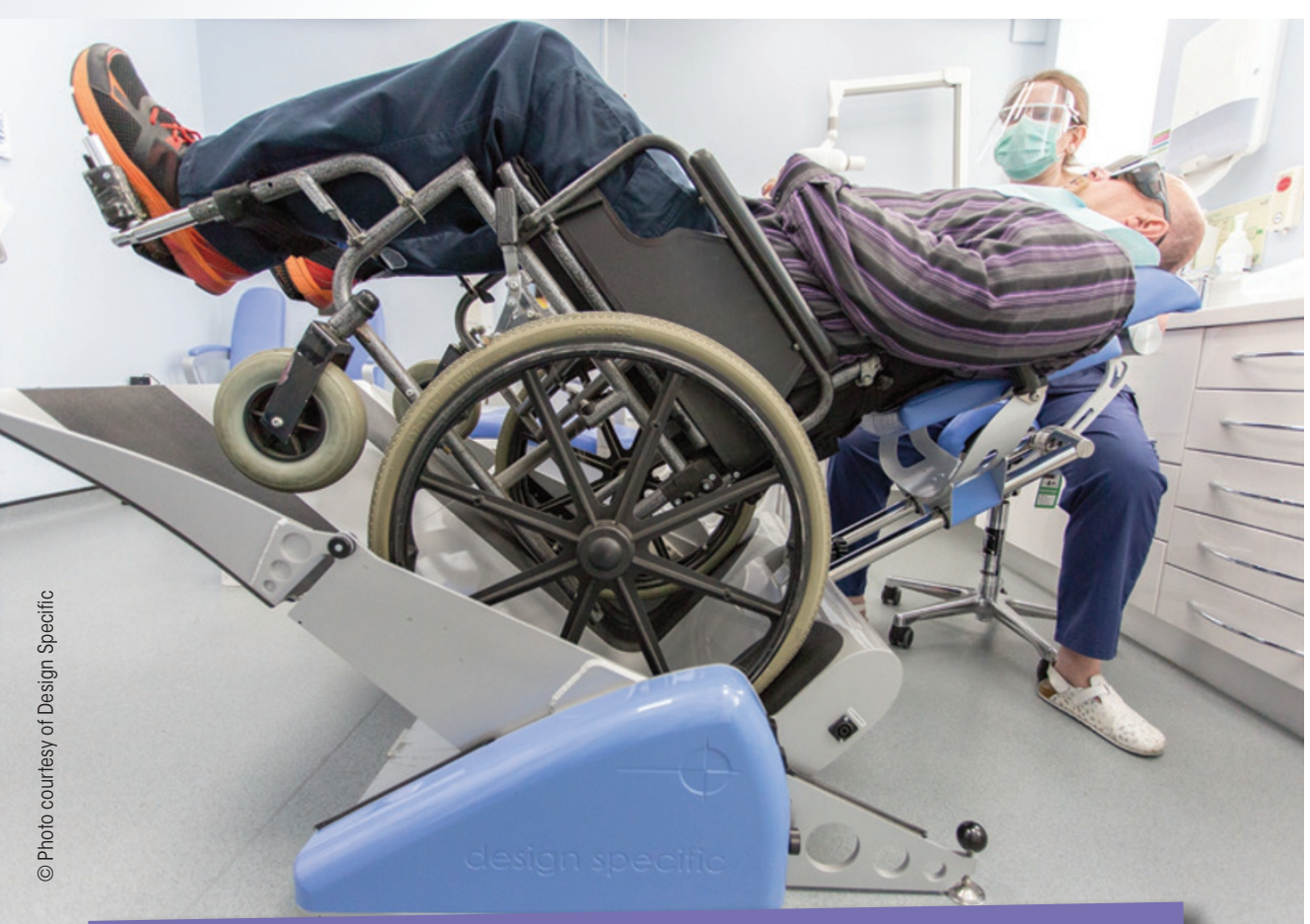

'COMMUNICATION AMONGST THE DENTAL

\section{TEAM IS VITAL'}

lack mental capacity and do not have an appropriate family member or friend to represent their views.

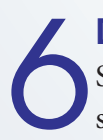
Distraction

Shifting the patient's attention to something less unpleasant than the procedure being carried out can help with most patients, for example, asking the patient having a local anaesthetic to wriggle their toes or talking to them about a favourite past-time or cartoon character.

This is something that the clinician and nurse can do together but is more commonly done by the dental nurse. Relatives or staff can help too by holding hands, chatting and comforting throughout. Some surgeries may have posters or coloured lighting on the ceiling which the patient can be asked to focus on.

\section{8} Oral hygiene advice

This is often a big struggle for carers and patients alike and the main thing that can really make a difference when done properly.

You will need to demonstrate to carers and parents the correct techniques. Encourage having two to brush, one who actually brushes and one who distracts the patient, holds hands and encourages them. Show them how to use an additional brush as a prop if the patient tends to clamp down.

If carers are struggling with compliance, brushing in a different room could help.

Be aware of products available such as grips for toothbrushes, Barman's 3 headed toothbrush and bite supports which could be suggested as brushing aids.
9 Cancer patients

All of the above still applies to this patient group but these patients must have additional support both pre- and posttreatment with oral hygiene, they must be monitored for signs and symptoms of oral mucositis to reduce the risk of aspiration pneumonia (see the WHO Oral Mucositis Scale), they may consider dental treatment and oral hygiene as their last priority so give as much information as possible as to reasons to keep up with mouth care plus lots of support and empathy.

There are products to assist with dry mouth, sore mouth and the inability to eat as a consequence of both, for example, a non-foaming (SLS free) and non-flavoured toothpaste or a mouthwash which acts as a 'liquid plaster' and enables the patient to eat in relative comfort.

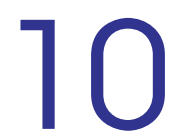
Communication is key Where possible, have a 'named nurse'. Someone the patient and their support network can ask to speak to who is familiar with their specific needs is important for continuity of care and can avoid repeat explanations on both sides.

Bear in mind it is good to be in contact with other disciplines when required, such as the GP for medications and medical history or health visitors and school nurses (especially if there are safe-guarding issues). If a patient fails their appointment do your best to find out why. Make phone calls to family members, where applicable to keep them informed of treatment and care.

Communication amongst the dental team is vital; it ensures all the valuable information gleaned previously as to how to assist the patient in accessing dental care and advice is utilised and available to all staff involved.

\section{Further reading}

Oral Mucositis ‘The OXO Mum's Sore Mouth', Emma Riley.

\section{References}

1. General Dental Council, Standards for the Dental Team http://www.gdc-uk.org.

2. National Institute of Dental and Craniofacial Research, Oral Complications of Cancer Treatment: What the Dental Team Can Do. www.nidcr.nih.gov/ oralhealth/Topics/CancerTreatment/ OralComplicationsCancerOral.htm

3. World Health Organisation. Handbook for reporting results of cancer treatment. World Health Organisation, Geneva, Switzerland, 1979: 15-22.

bdjteam2015152 